\title{
Early Results from DOE/NREL Transit Bus Evaluations
}

Leslie Eudy

\author{
NREL
}

Kevin Chandler

\author{
Battelle
}

\section{APTA Bus and Paratransit Conference}

Columbus, Ohio

$$
\text { May 17, } 2005
$$




\section{Overview}

- Background

- Early Results from New York City Transit

- Early Results from King County Metro

- Questions 


\section{NREL Fleet Test \& Evaluation Team}

- Evaluate "real world" performance of advanced propulsion technologies

- Focus on medium and heavy duty fleet applications

- Main goals:

- Facilitate the transition of advanced technologies from the R\&D stage into the marketplace

- Provide potential fleet users with accurate and unbiased information on vehicle performance and costs

- Funding is provided by the Department of Energy's Office of Energy Efficiency and Renewable Energy

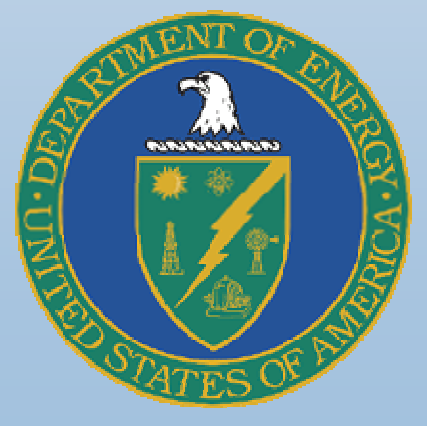




\section{Fleet Evaluation: Goals}

- Objectives:

- Assess status of new technologies in real-world service

- Establish a benchmark of current and near-term technologies

- Provide unbiased information on vehicle performance

- Share lessons learned about implementation experience

- Target Audience:

- Fleet managers considering use of the technology

- Manufacturers \& system integrators

- Department of Energy

- Other interested parties

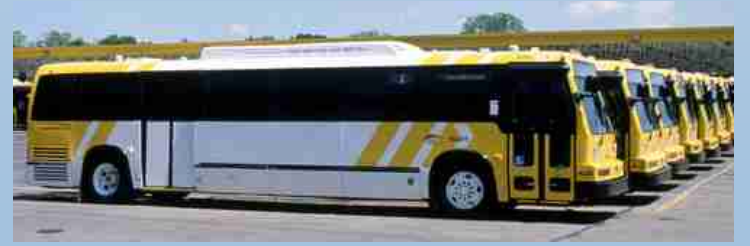

(APTA, FTA, EPA, EDTA...) 


\section{Fleet Evaluation: Specifics}

Selection of Specific Technology and Site for

Evaluation

- Fuel and Technology Neutral

- Alternative fuel - CNG, LNG, Biodiesel, LPG

- Electric propulsion - hybrid-electric, fuel cell

- Site Selection Criteria include:

- At least 5 advanced technology vehicles

- Good record keeping

- Motivated to participate 


\section{Fleet Evaluation: Specifics}

Data collection plan based on existing and proven protocol developed for DOE heavy vehicle evaluations.

Data Collection includes:

- Vehicle specifications

- Vehicle expectations

- Vehicle usage and specific duty cycle

- Fuel and oil consumption

- Maintenance

- Fleet implementation experience

- Facility descriptions and capital cost

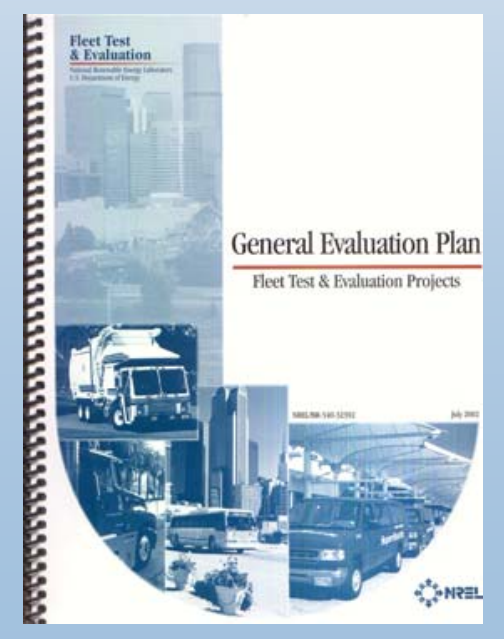




\section{Fleet Evaluation: Specifics}

Analysis and Reporting

The data collection typically covers one year of operation for both advanced vehicles and conventional comparison (if available).

Reports include:

- Two page fact sheet

- Early experience and results report

$\mathrm{O}=$

SunLine Test Drives Hydrogen Bus

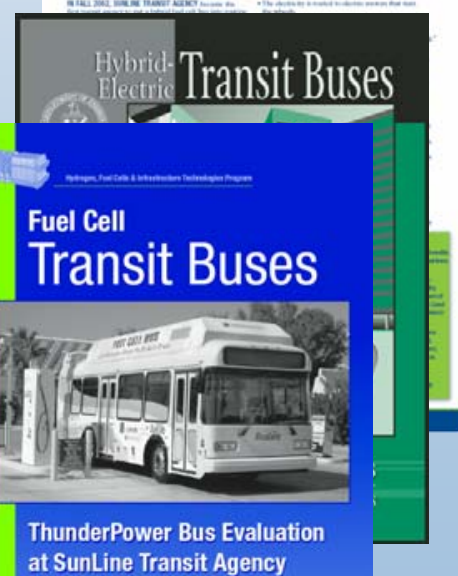
at SunLine Transit Agency

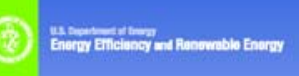

- Final Summary Report 


\title{
Early Hybrid Bus Experience at NYCT in New York City
}

\author{
Leslie Eudy \\ National Renewable Energy Laboratory
}




\section{Agenda}

- Quick NYCT clean bus program description

- Plans for DOE/NREL evaluation

- Hybrid, Diesel, and CNG bus descriptions

- Early experience

- What's next 


\section{NYCT Clean Bus Program}

NYCT Goals

- Reduce bus fleet emissions

- Improve service (reliability and noise)

- Reduce cost of operations (improve fuel economy and reduce costs) 


\section{NYCT Clean Bus Program}

- Repowering old 2-stroke diesel engines to new EGR-equipped 4-stroke diesel engines; to be completed in 2005

- All diesel engines to be equipped with diesel particulate filters (in progress)

- CNG buses - 481 in operation

- Hybrid buses - 325 in operation by mid2005 


\section{NYCT Reasons for Hybrid Buses}

- Emissions reductions

- Increased fuel economy

- Smooth and quiet operation

- Improved performance

- Avoids infrastructure costs of CNG 


\section{CNG and Hybrid Buses}

\begin{tabular}{|l|c|c|c|}
\hline Depot & CNG (260) & Hybrid (125) & Hybrid (200) \\
\hline Gleason & 95 & & \\
\hline West Farms & 165 & & \\
\hline $\begin{array}{l}\text { Mother Clara } \\
\text { Hale }\end{array}$ & & 60 & \\
\hline $\begin{array}{l}\text { Queens } \\
\text { Village }\end{array}$ & & & 135 \\
\hline Fresh Pond & & & 65 \\
\hline $\begin{array}{l}\text { Manhattan- } \\
\text { ville }\end{array}$ & & & \\
\hline
\end{tabular}




\section{Planned DOE/NREL Evaluation}

- Hybrid Order of 125 Orion VII Buses - 10 Hybrid Buses at Mother Clara Hale Depot in Manhattan Division (10 Orion V diesel buses chosen for comparison) Average speed at depot of $6.5 \mathrm{mph}$
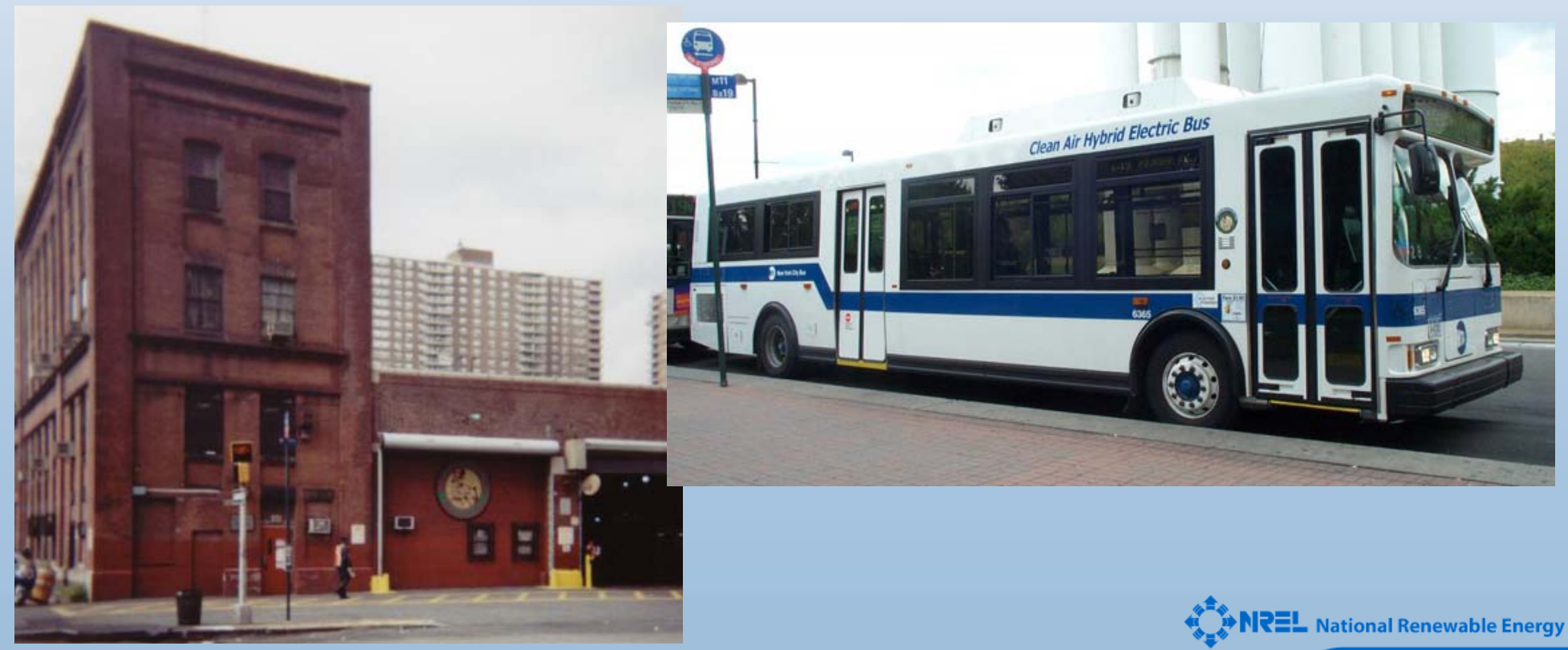


\section{Planned DOE/NREL Evaluation}

- CNG Order of Orion VII Buses - 10 CNG Buses at West Farms Depot in Bronx Division (10 NovaBus RTS diesel buses chosen for comparison) - Average speed at depot of 6.5 $\mathrm{mph}$

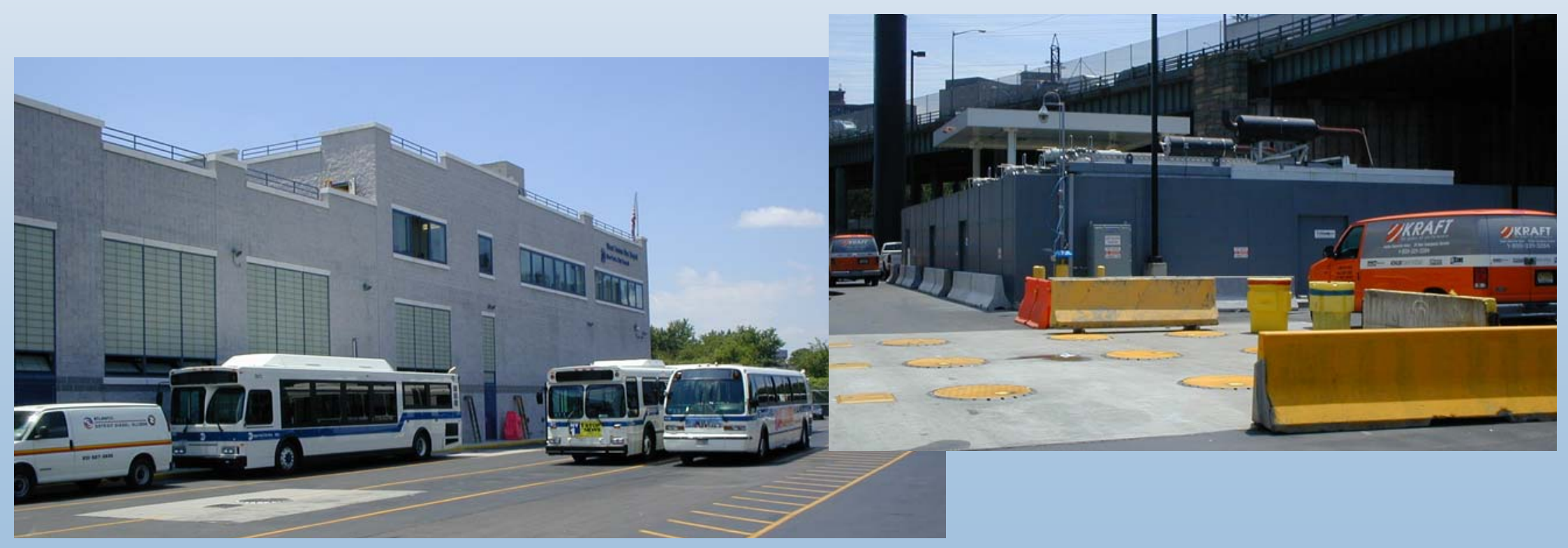




\section{Planned DOE/NREL Evaluation}

- Hybrid Order of 200 Orion VII Buses - 10 Hybrid Buses at Fresh Pond Depot (10 diesel buses to be chosen for comparison)
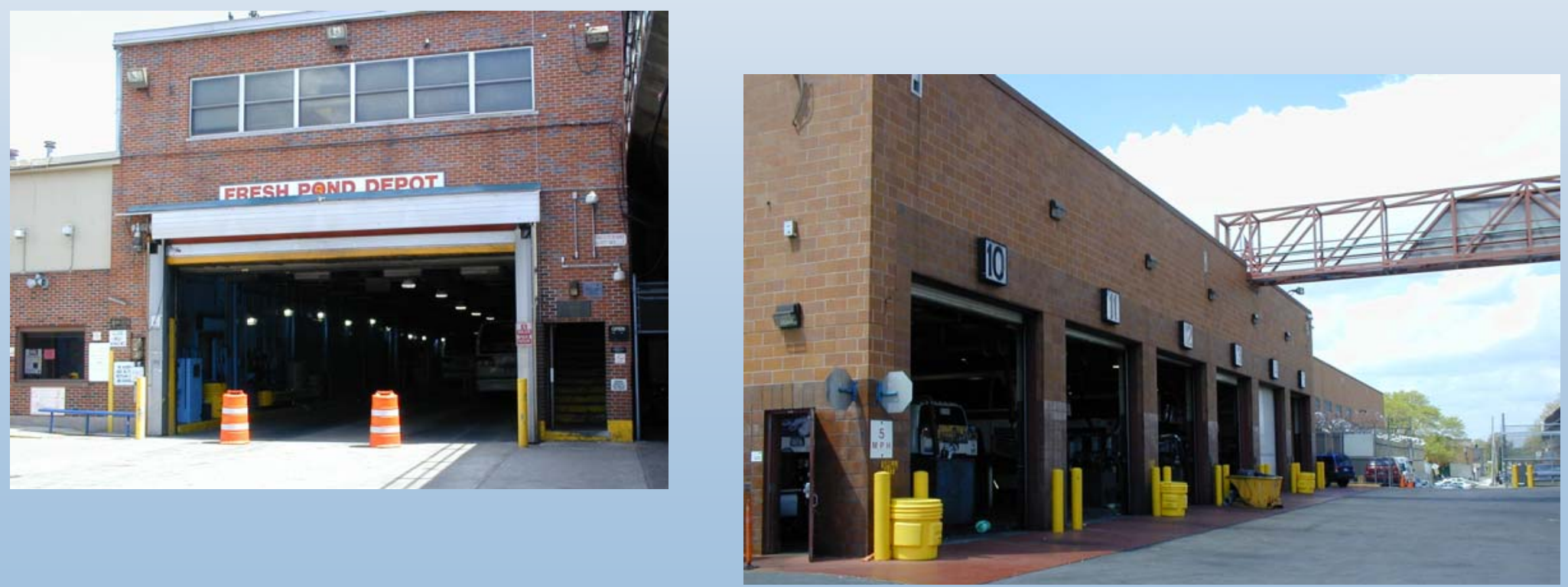


\section{Planned DOE/NREL Evaluation}

- Collect and analyze operations data (bus usage, fuel, and maintenance) for 12 months

- Order of 125 Hybrid buses and CNG bus evaluations starting with October 2004

- Hybrid Order of 200 evaluation just getting underway 


\section{Vehicle Descriptions}

\begin{tabular}{|l|l|l|}
\hline System & Hybrid (125) & CNG \\
\hline Bus & Orion VII 40’ & Orion VII 40’ \\
\hline Engine & Cummins ISB & DDC S50G \\
& $270 \mathrm{hp}$ & $275 \mathrm{hp}$ \\
& $660 \mathrm{ft}-\mathrm{lb}$ & $900 \mathrm{ft}-\mathrm{lb}$ \\
\hline Emissions & $4.0 \mathrm{~g} / \mathrm{bhp}-\mathrm{hr}$ & $2.5 \mathrm{~g} / \mathrm{bhp}-\mathrm{hr}$ \\
& NOx; 0.05 & NOx+HC; 0.05 \\
& g/bhp-hr PM & g/bhp-hr PM \\
& using a DPF & without Cat \\
\hline
\end{tabular}




\section{Vehicle Descriptions}

\begin{tabular}{|l|l|l|}
\hline System & Hybrid (125) & CNG \\
\hline $\begin{array}{l}\text { Electric } \\
\text { Propulsion }\end{array}$ & $\begin{array}{l}\text { BAE SYSTEMS } \\
\text { HybriDrive }^{T M}\end{array}$ & None \\
\hline Energy Storage & $\begin{array}{l}\text { Sealed lead } \\
\text { acid }\end{array}$ & None \\
\hline $\begin{array}{l}\text { Regenerative } \\
\text { Braking }\end{array}$ & Yes & None \\
\hline
\end{tabular}




\section{Early Experience}

- CNG Orion VII buses started arriving at NYCT in March 2003

- West Farms started getting their first CNG Orion VII buses in September 2003

- Hybrid buses started operation at Mother Clara Hale Depot in March 2004

- Hybrid buses started operation at Queens Village Depot in May 2004 


\section{Average Monthly Mileage per Bus CNG Buses}

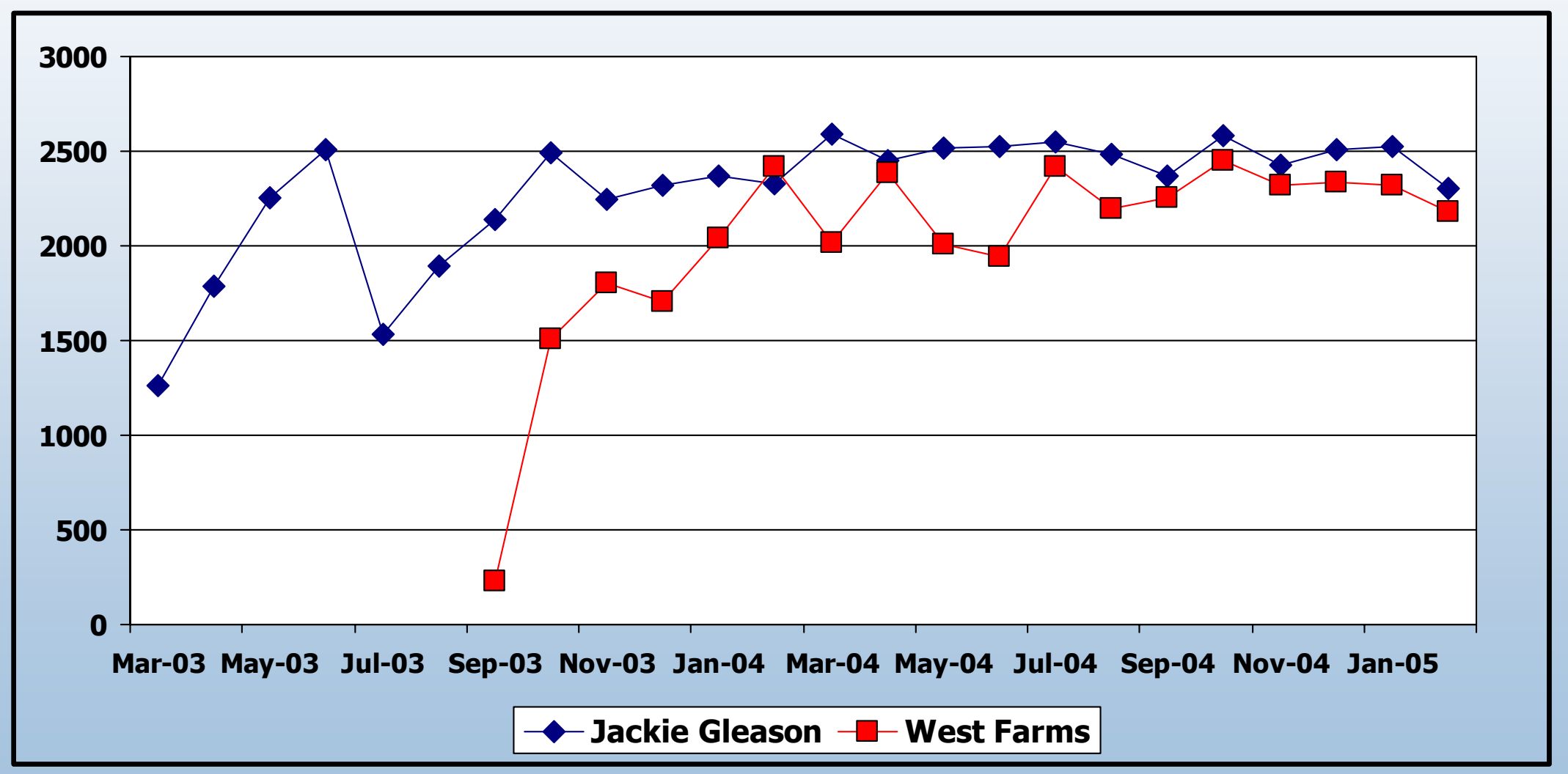




\section{Average Monthly Mileage per Bus Hybrid Buses}

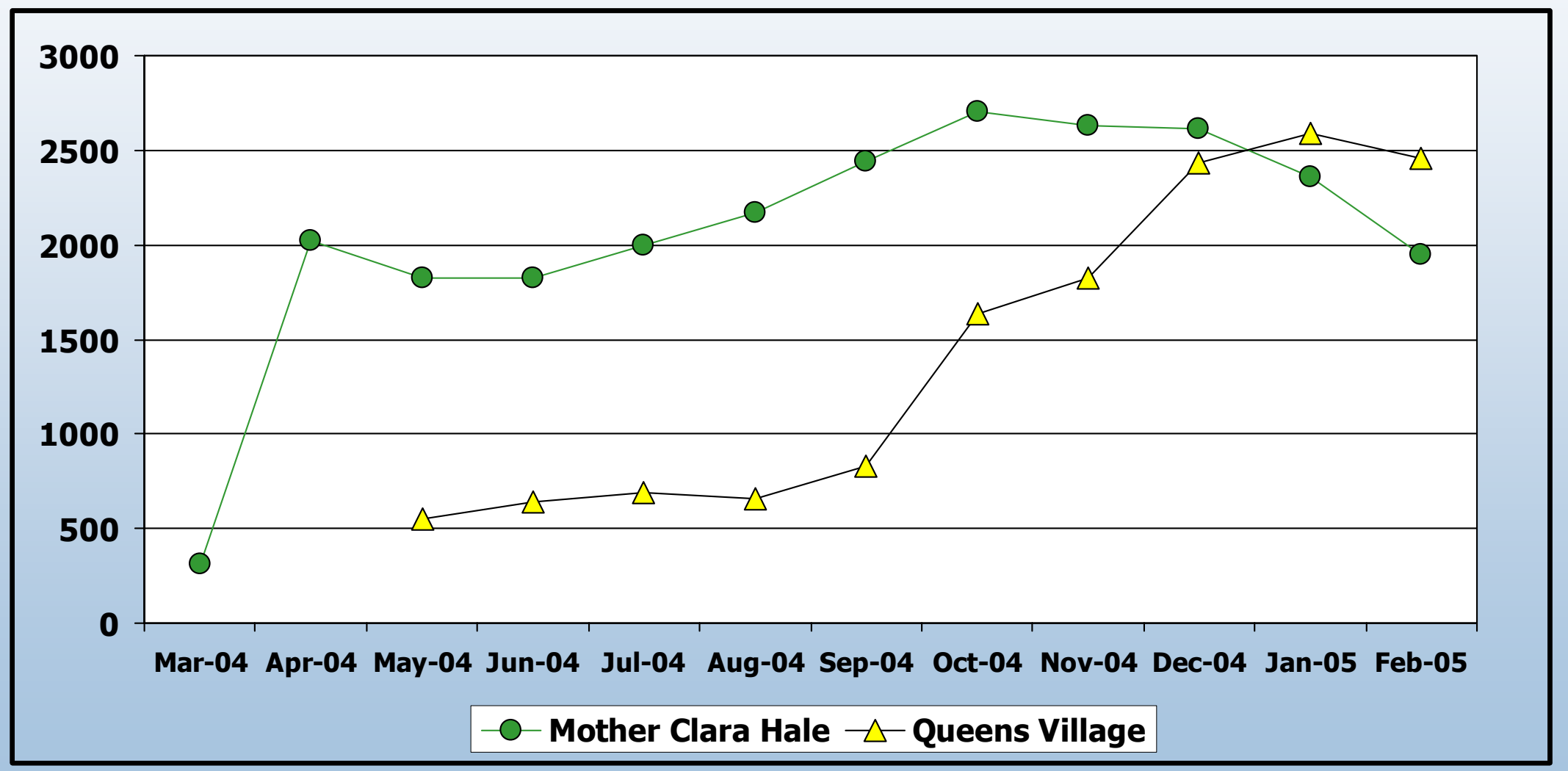




\section{Miles Between Roadcalls (MBRC) CNG Buses}

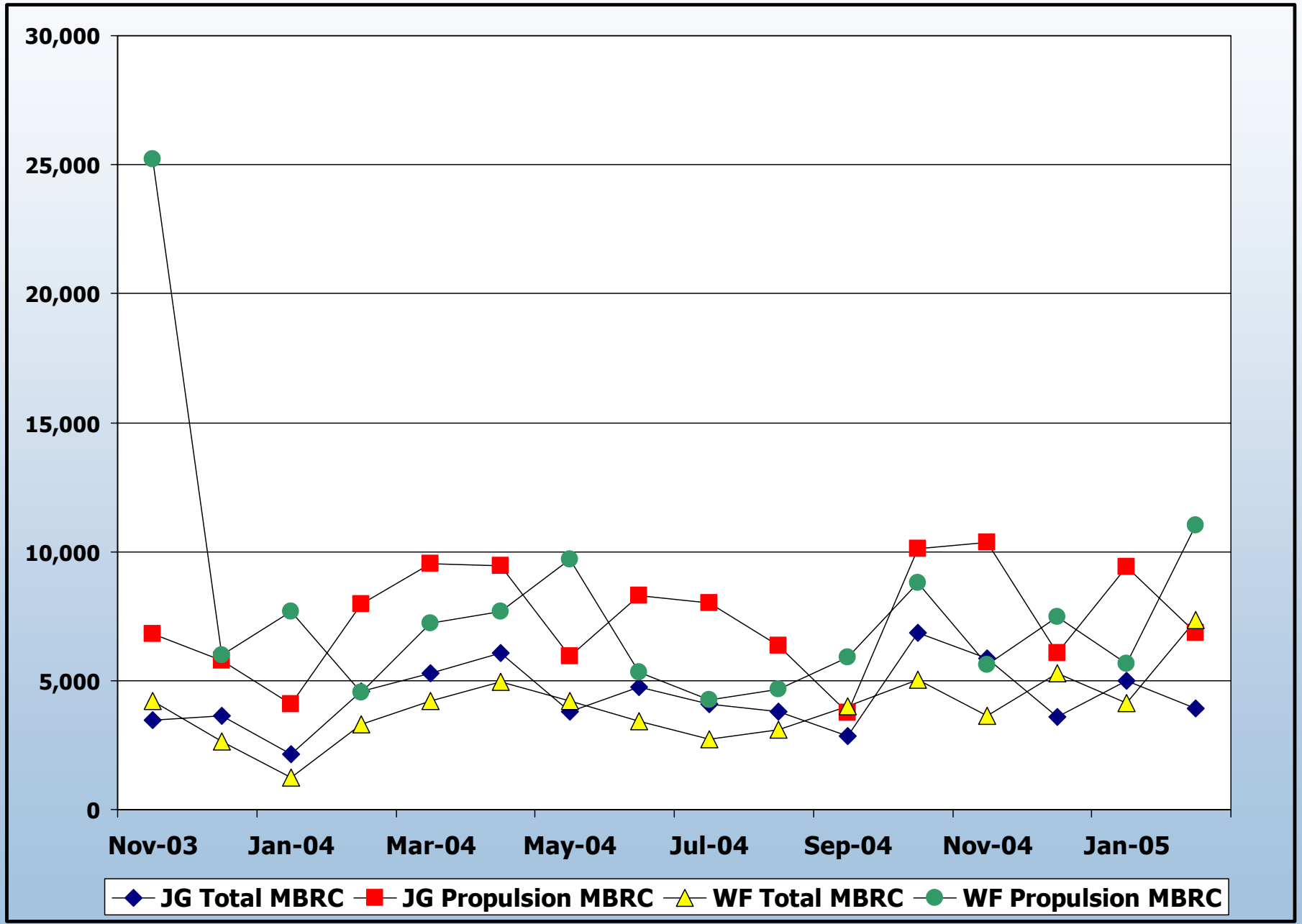




\section{Miles Between Roadcalls (MBRC) Hybrid Buses}

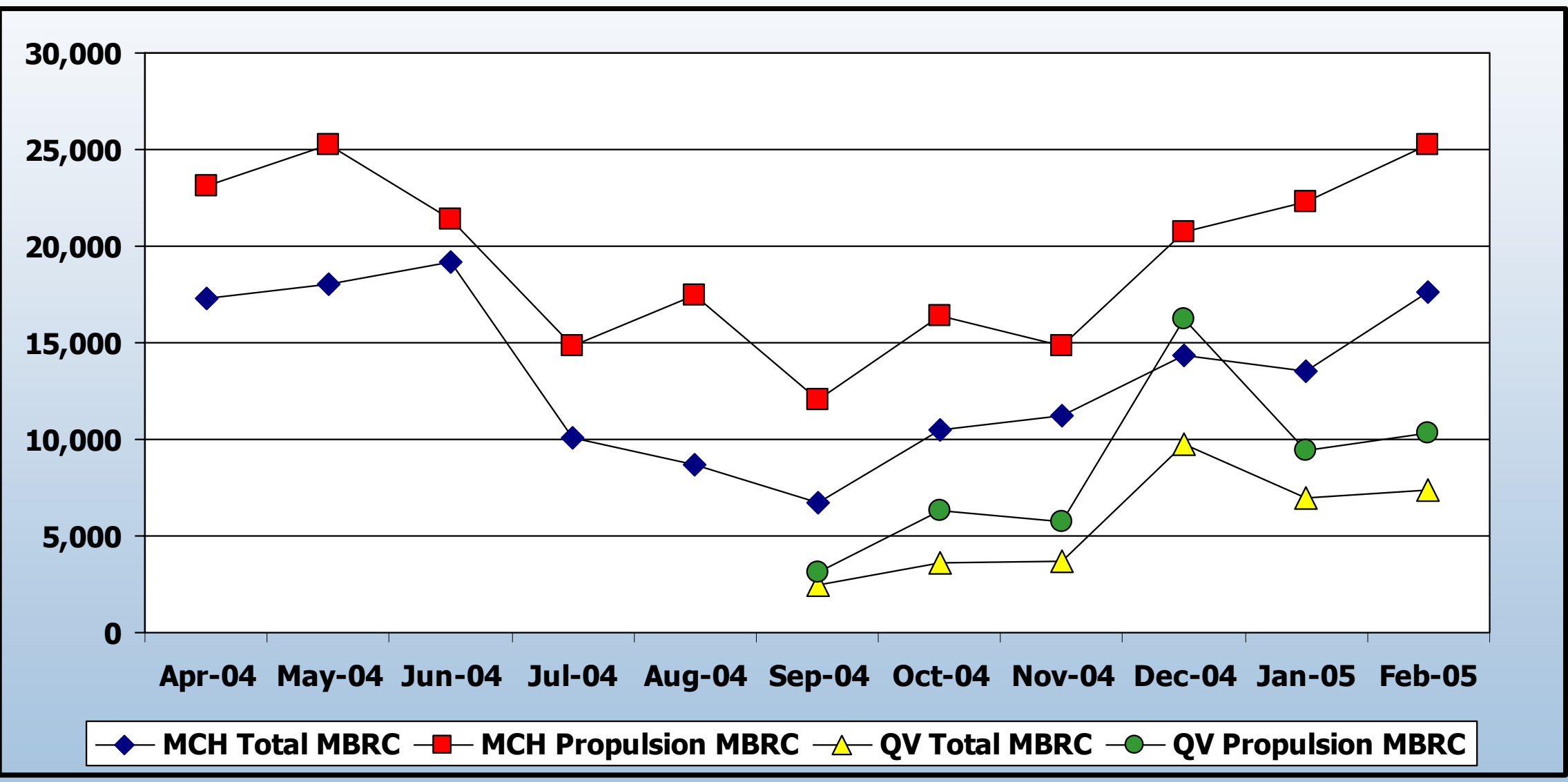




\section{Fleet Campaigns - Orion VII CNG and Hybrid}

\section{Bus}

- Axle bolts coming loose

- Radiator baffle, surge tank overflowing

- Fuel lift pump

- Fuel injection pump

- PCS cooling pump seizure

- Water in wiring/connectors

- Engine grid heater relay

- Water in engine from air intake

Hybrid

- PCS internal coolant leaks

- PCS board modification

- Software upgrade

\section{CNG}

- Cylinder kits

- Spark plugs 


\section{Early Results for Evaluation Buses}

- Data period for results that follow:

- West Farms Depot, 10/04-3/05

- Mother Clara Hale Depot, 10/04-3/05

- The following information represents early experience, NOT final results 


\section{Early Results for Evaluation Buses Fuel Economy - CNG and Diesel}

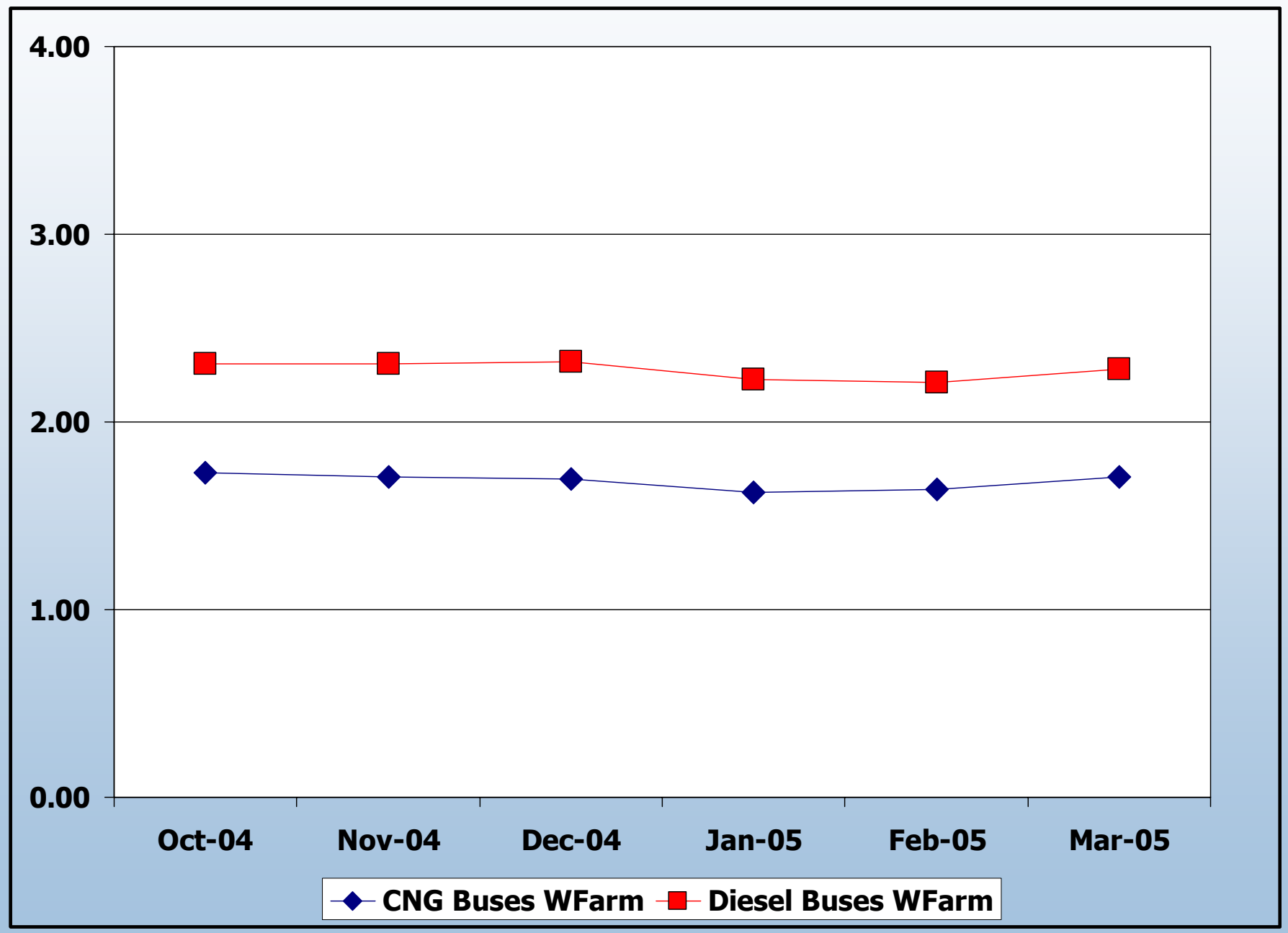




\section{Early Results for Evaluation Buses Fuel Economy - Hybrid and Diesel}

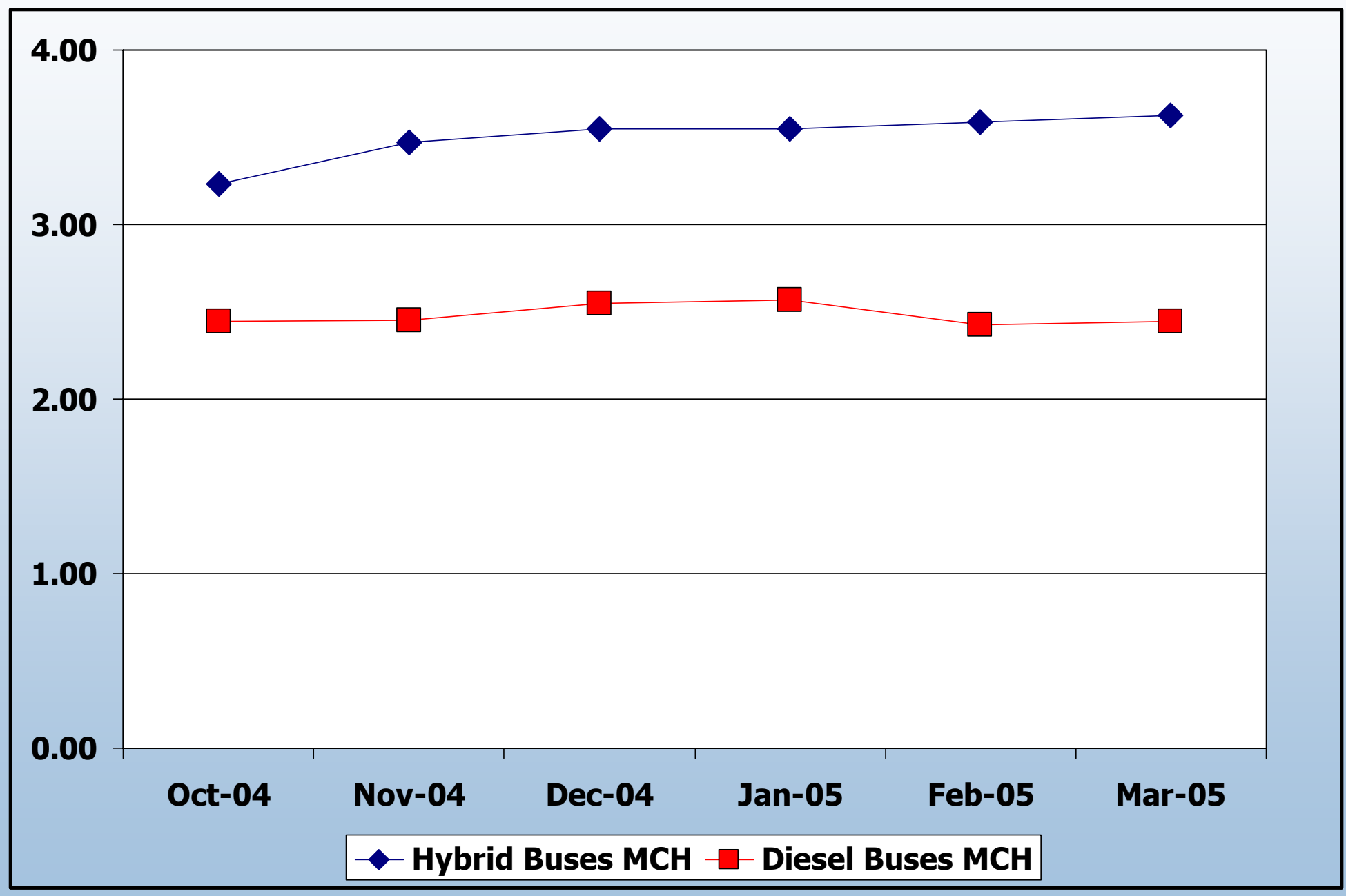




\section{What's Next}

- Complete DOE/NREL evaluation - 12 months of operations for the Hybrid order of 125 and CNG buses starting October 2004

- Evaluation of Hybrid order of 200 just getting under way

- Reports

- Fact sheet available

- Interim report planned for Fall of 2005

- Final report planned for mid-2006 


\section{Special Thanks}

- New York City Transit

- Orion Bus

- BAE SYSTEMS

- U.S. Department of Energy 


\section{Early Hybrid Bus Experience at KC Metro in Seattle, WA}

\section{Kevin Chandler Battelle}




\section{Agenda}

- Quick KC Metro hybrid program description

- Hybrid and Diesel bus descriptions

- Early experience

- Plans for DOE/NREL evaluation

- What's next 


\section{King County Metro Hybrid Bus Program}

- Purchased 235 New Flyer/Allison Electric Drive articulated buses (October 2003) - $\$ 645,000$ each

- Hybrid buses replacement for Breda dual-mode buses being retired

- Also purchased 30 diesel New Flyer articulated buses at the same time - $\$ 445,000$ each

- Sound Transit received 22 hybrid (of the 235 buses) and 16 diesel articulated buses, all operated by KC Metro 


\section{Vehicle Descriptions}

\begin{tabular}{|l|l|l|}
\hline System & Diesel & Hybrid \\
\hline Bus & New Flyer 60' & New Flyer 60' \\
\hline Engine & Caterpillar C9 & Caterpillar C9 \\
& $330 \mathrm{hp}$ & $330 \mathrm{hp}$ \\
& $1150 \mathrm{ft}-\mathrm{lb}$ & $1150 \mathrm{ft}-\mathrm{lb}$ \\
\hline Emissions & 2.5 g/bhp-hr & $2.5 \mathrm{~g} / \mathrm{bhp}-\mathrm{hr}$ \\
& NOx+HC; 0.05 & NOx+HC; 0.05 \\
& g/bhp-hr PM & g/bhp-hr PM \\
& using a DPF & using a DPF \\
\hline
\end{tabular}




\section{Vehicle Descriptions}

\begin{tabular}{|l|l|l|}
\hline System & Diesel & Hybrid \\
\hline $\begin{array}{l}\text { Electric } \\
\text { Propulsion }\end{array}$ & None & $\begin{array}{l}\text { Allison EP50 } \\
\text { Parallel Hybrid } \\
\text { System }\end{array}$ \\
\hline $\begin{array}{l}\text { Energy } \\
\text { Storage }\end{array}$ & None & $\begin{array}{l}\text { Nickel metal } \\
\text { hydride } \\
\text { batteries }\end{array}$ \\
\hline $\begin{array}{l}\text { Regenerative } \\
\text { Braking }\end{array}$ & None & Yes \\
\hline
\end{tabular}




\section{New Flyer Articulated Buses at KC Metro}
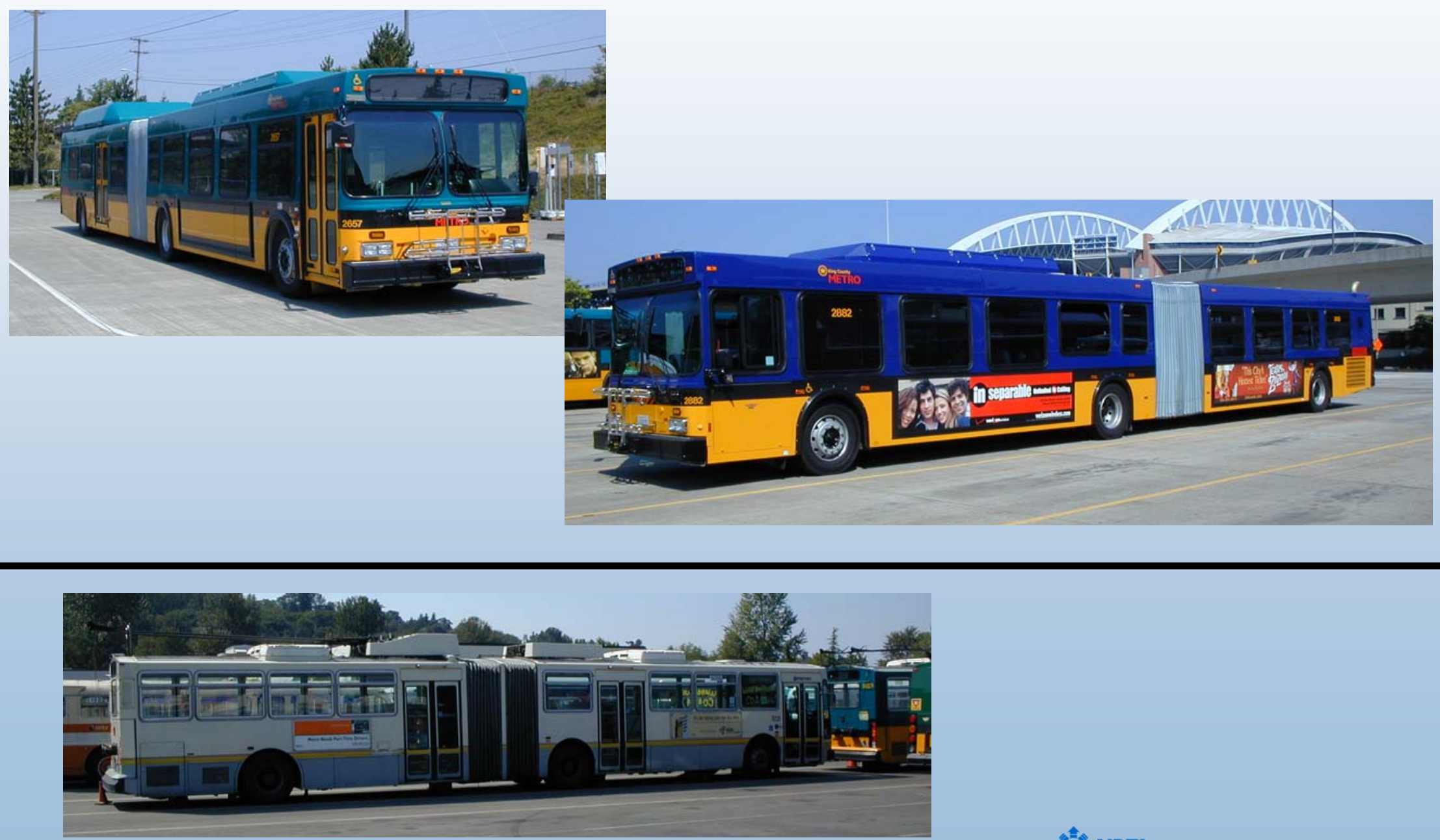


\section{Early Experience}

- Introduction of 235 hybrid buses started in June 2004 and was completed by December 2004

- Hybrid buses reported as easy to put into service within 2-3 days after delivery

- By end of February 2005, fleet have completed $\sim 4$ million miles ( $\sim 6$ million miles as of today) 


\section{Average Monthly Mileage per Bus}

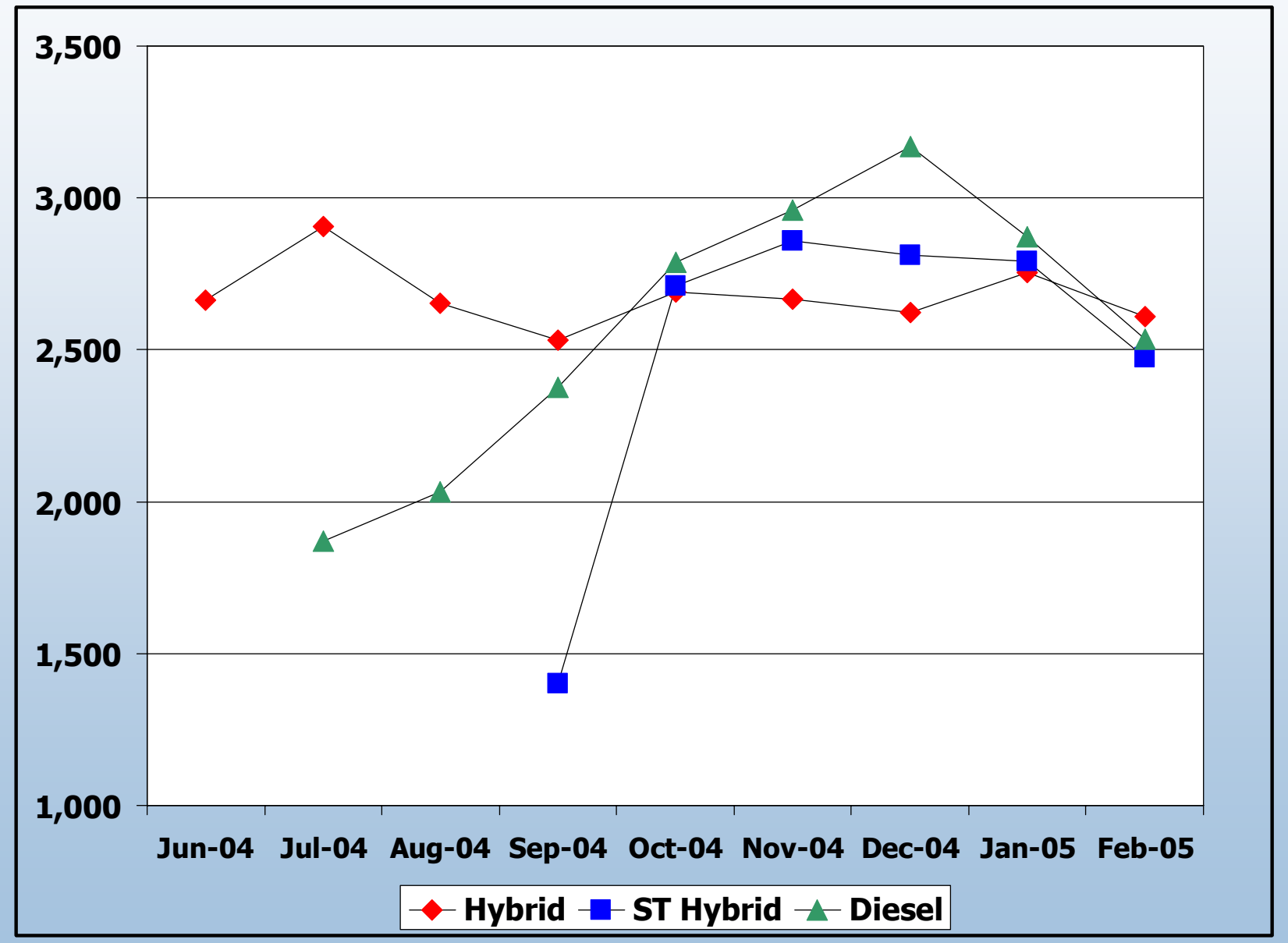




\section{Roadcalls}

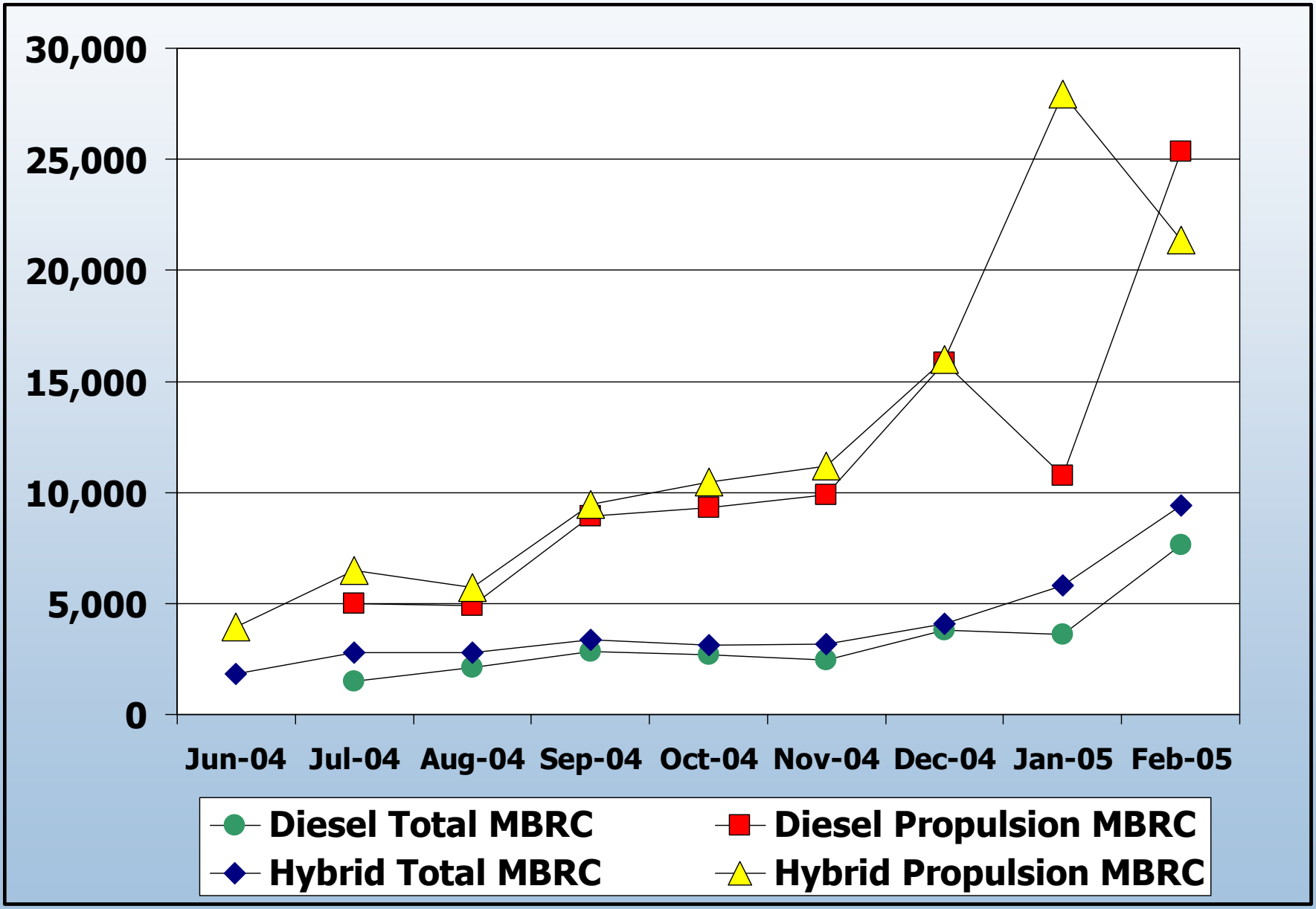




\section{Fleet Campaigns}

- Axle snap rings - 8/04

- Soot filter replacement - 8/04

- Communication modules - 8/04

- Allison/CAT software upgrade - 9/04

- Door proximity switches - 10/04

- Center axle radius rod bolts - 11/04

- Wire chaffing over rear door - 12/04

- Master switch - 12/04

- Mirror drain holes - 1/05 


\section{Planned DOE/NREL Evaluation}

- 10 Hybrid Buses at Atlantic Base (best match in operation compared to diesel buses) - Average speed of $11.2 \mathrm{mph}$

- 10 Hybrid Buses at South Base (first base to put hybrids into service) - Average speed of 19.2 mph

- 10 Diesel Buses at Ryerson Base - Average speed of $13.3 \mathrm{mph}$

- Collect and analyze operations data (bus usage, fuel, and maintenance) for 12 months starting with April 2005 


\section{Early Results for Evaluation Buses}

- Data period for results that follow:

- South Base Hybrids, 7/04-2/05

- Atlantic Base Hybrids, 12/04-2/05

- Ryerson Base Diesels, 7/04-2/05

- The following information represents early experience, NOT final results 


\section{Early Results for Evaluation Buses Fuel Economy}

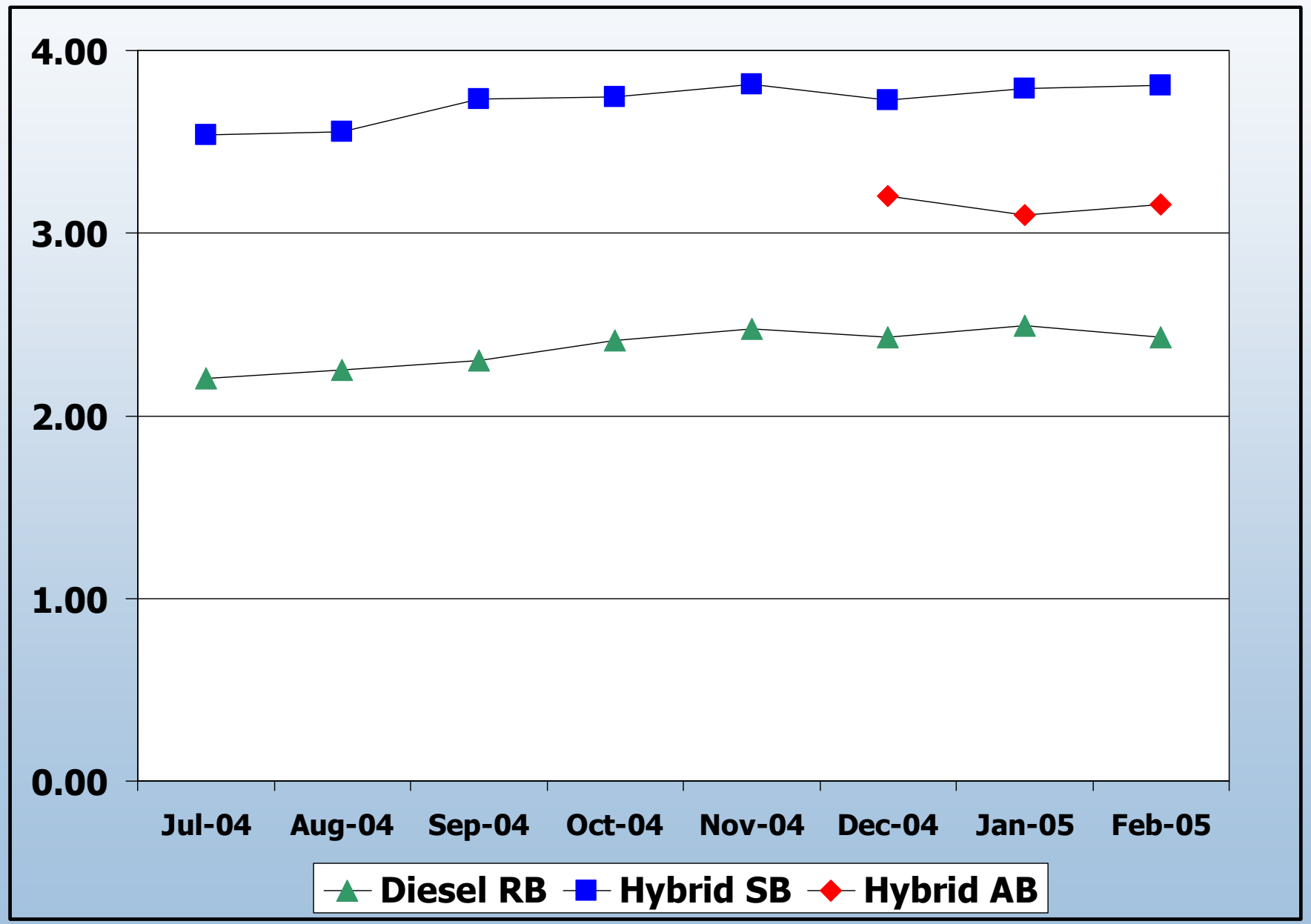




\section{Early Results for Evaluation Buses Maintenance Costs per Mile}

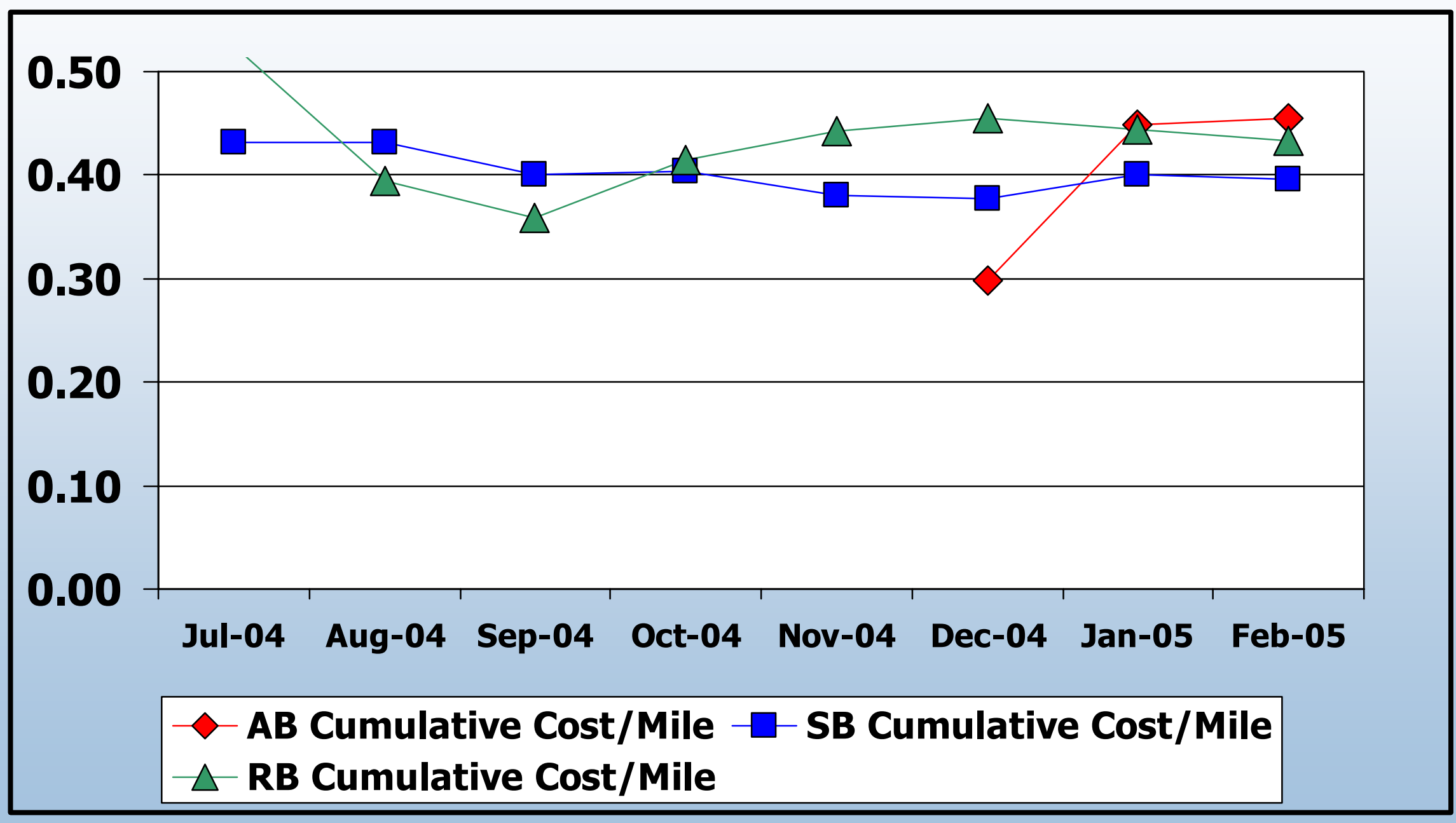




\section{Early Results for Evaluation Buses Fuel and Maintenance Cost/Mile}

\begin{tabular}{|l|l|l|l|}
\hline & Diesel RB & Hybrid AB & Hybrid SB \\
\hline $\begin{array}{l}\text { Fuel } \\
\text { (\$1.47/gal) }\end{array}$ & $\begin{array}{l}\$ 0.614 / \mathrm{mi} \\
\text { Maintenance }\end{array}$ & $\$ 0.466 / \mathrm{mi}$ & $\$ 0.396 / \mathrm{mi}$ \\
& & $3.15 \mathrm{mpg}$ & $3.72 \mathrm{mpg}$ \\
\hline Total & $\$ 1.047 / \mathrm{mi}$ & $\$ 0.456 / \mathrm{mi}$ & $\$ 0.396 / \mathrm{mi}$ \\
& & & \\
\hline
\end{tabular}




\section{What's Next}

- Complete DOE/NREL evaluation - 12 months of operations for the 30 study buses starting April 2005

- Chassis Dynamometer Emissions Testing

- Reports

- Fact sheet available

- Interim report planned for end of 2005

- Final report planned for mid-2006 


\section{Special Thanks}

- King County Metro

- New Flyer

- Allison Electric Drives

- Caterpillar

- U.S. Department of Energy 